Supporting Information

\title{
Enhanced Piezoelectric Performance of various Electrospun PVDF Nanofibers and Related Self-power Devices Applications
}

Shuangzhe Zhang ${ }^{1,2 \dagger}$, Bowen Zhangl, ${ }^{1, \dagger}$, Jinxi Zhang ${ }^{1}$ and Kailiang Ren ${ }^{1,4^{*}}$

${ }^{1}$ CAS Center for Excellence in Nanoscience, Beijing Key Laboratory of Micro-nano Energy and Sensor, Beijing Institute of Nanoenergy and Nanosystems, Chinese Academy of Sciences, Beijing, 101400, P. R. China

${ }^{2}$ School of Nanoscience and Technology, University of Chinese Academy of Sciences, Beijing 100049, P. R. China

${ }^{3}$ School of Physical Sciences, University of Chinese Academy of Sciences, Beijing 100049, P. R. China

${ }^{4}$ Research Center for Optoelectronic Materials and Devices, School of Physical Science and Technology, Guangxi University, Nanning 530004, China

$\dagger$ These authors made equal contributions to this work.

${ }^{*}$ Correspondence to: kailiang ren: renkailiang@binn.cas.cn 


\section{Supporting Figures}
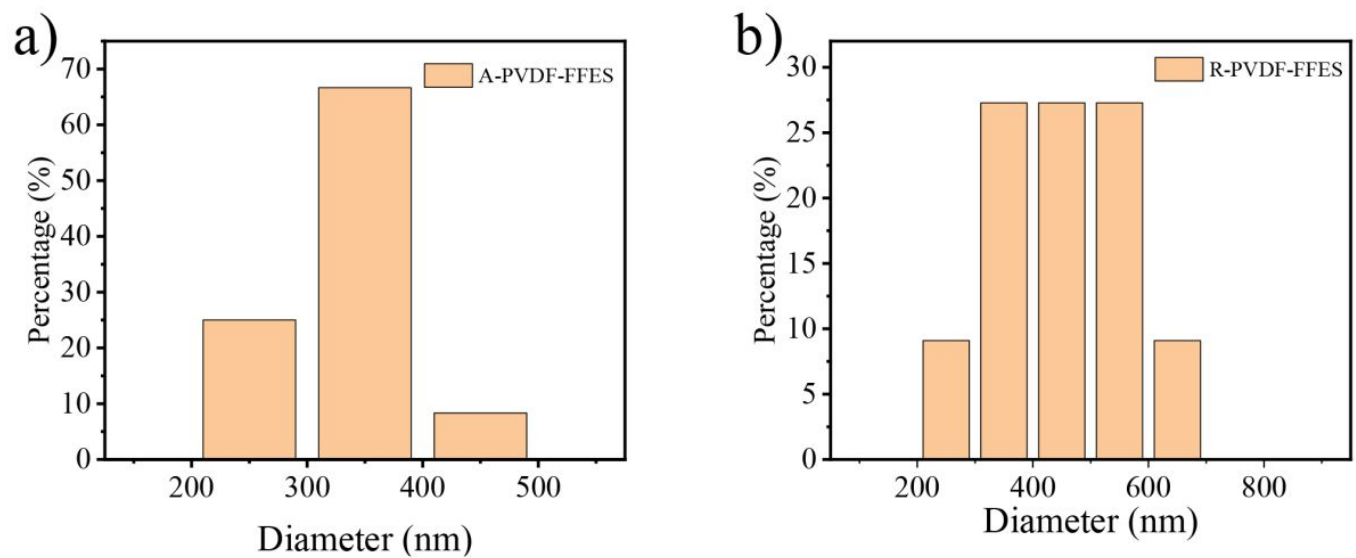

Figure S1. Diameter distribution of (a) A-PVDF-FFES nanofibers (b) R- PVDF-FFES nanofibers. 

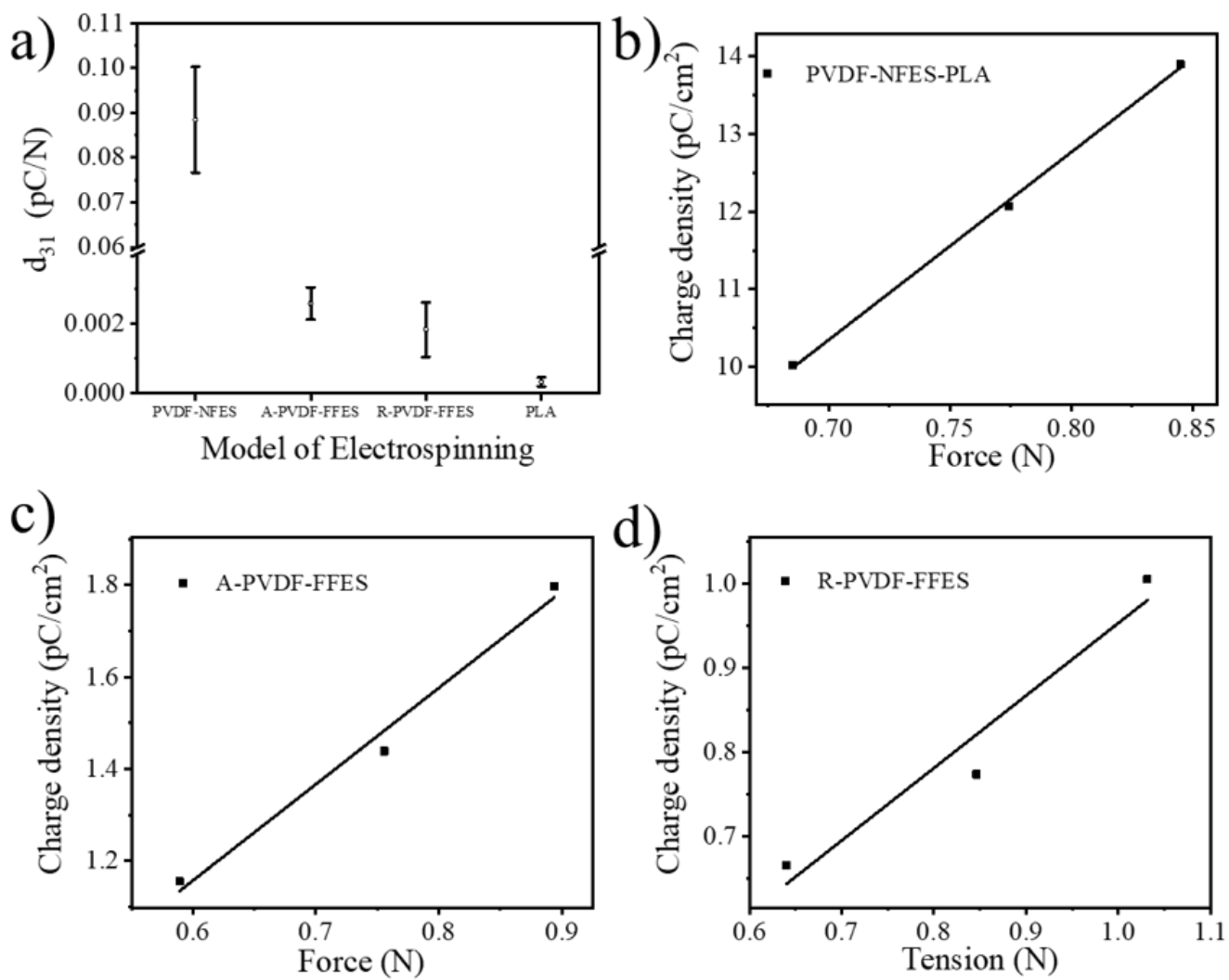

Figure S2. (a) Piezoelectric coefficient $d_{31}$ coefficient of PVDF-NFES-PLA film, APVDF-FFES nanofibers, R-PVDF-FFES nanofibers and PLA film. Charge density as a function of applied force of (b) PVDF-NFES-PLA film, (c) A-PVDF-FFES nanofibers, and (d) R-PVDF-FFES nanofibers. 


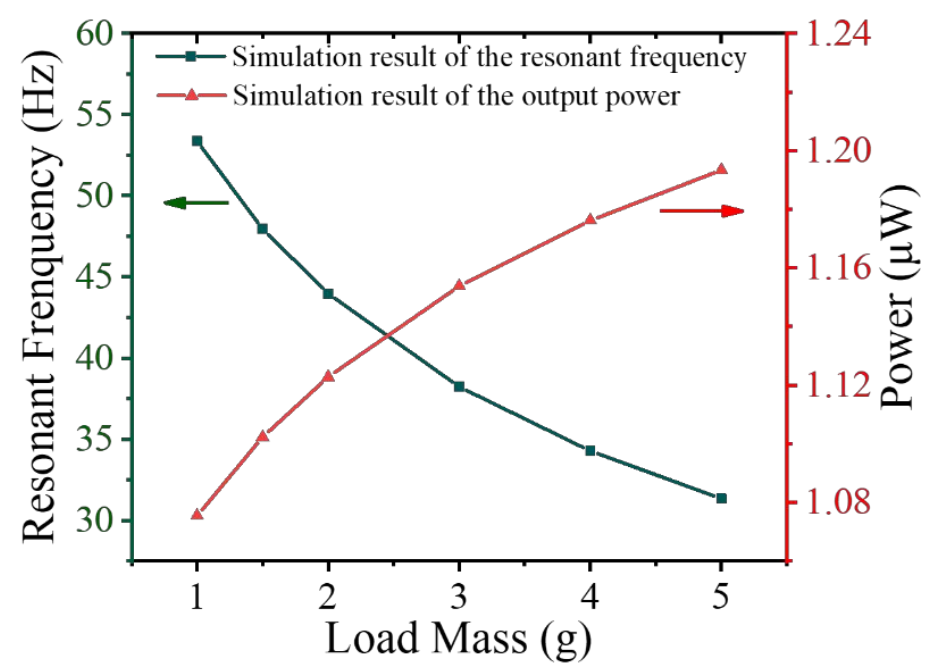

Figure S3. Simulation results of the power and resonance frequency as a function of loading mass. The simulation parameters include three parts: the substrate size, the mass and the piezoelectric layer. The size of substrate in this simulation is $15 * 10 * 1$ mm PET set on a $15 * 5 * 3 \mathrm{~mm}$ PET base. The mass is a $15 * 5 * 5 \mathrm{~mm}$ PTFE cuboid set on a side of PET substrate. The piezoelectric layer includes 14 indenpend PVDF fibers $(0.2 * 26.5 * 0.1 \mathrm{~mm}$ for each fibers $)$. In the simulation process, by applying different vibration frequencies to the model, the resonance frequency and output signal of the structure are detected. Then by changing the mass, the resonance frequency and maximum output under different masses can be obtained. 

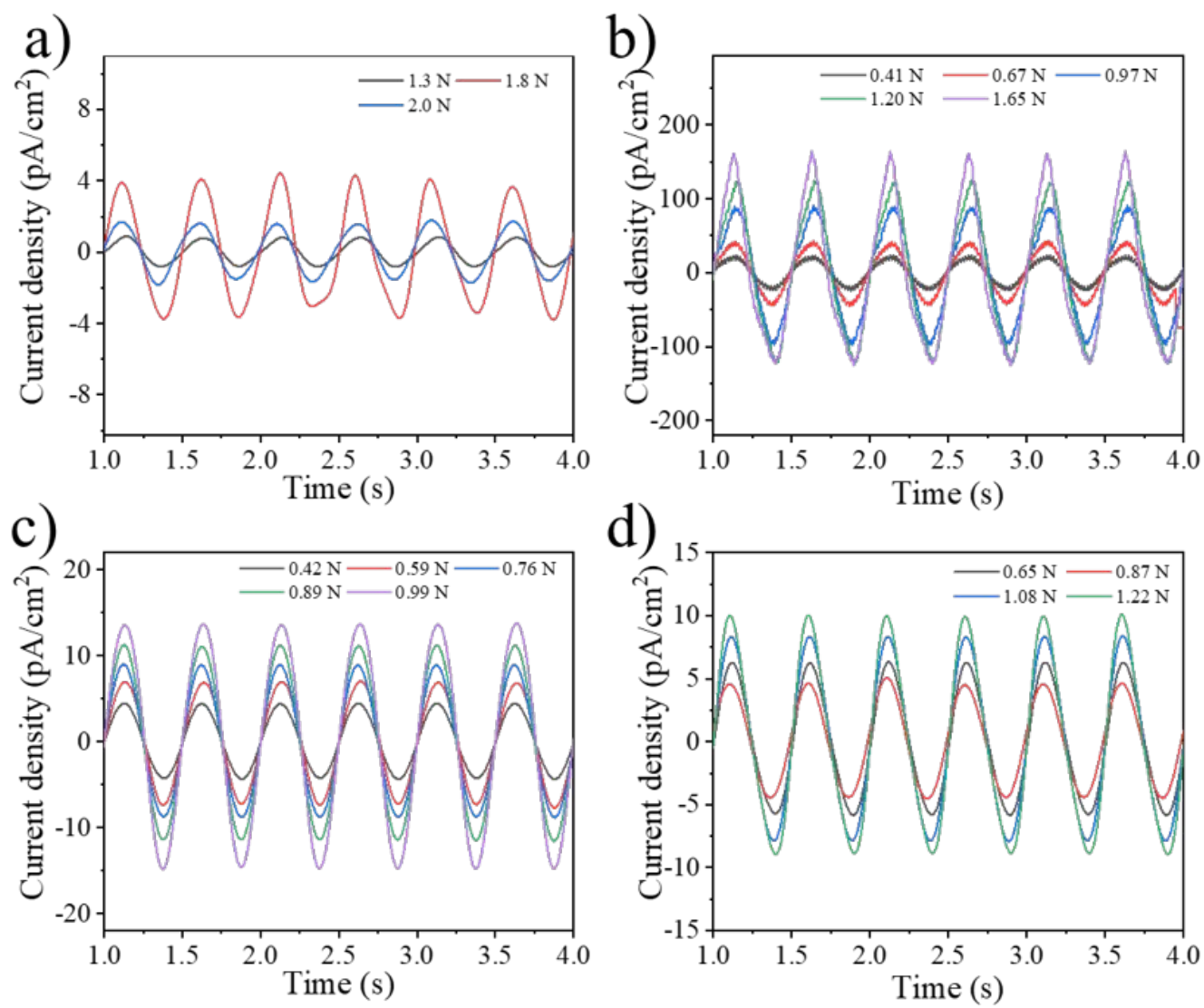

Figure S4. The current density in $\mathrm{d}_{31}$ output testing (a) PLA. (b) PVDF-NFES-PLA. (c) A-PVDF-FFES. (d) R-PVDF-FFES. 